\title{
Focus on the external auditory canal
}

The external auditory canal is a frequently neglected area of otology, with the middle and inner ears attracting more attention. However, this site, being where the skin and auditory organs meet, has some unique features of considerable interest. The important role of the external auditory canal, and some of the clinical issues relevant to it, are highlighted in this issue of the JLO.

From a practical standpoint, the commonest condition affecting the external auditory canal is accumulation of cerumen, or ear wax. This is a huge problem in both primary and secondary care and is associated with an industry solely concerned with cerumen removal and dispersal. Many preparations are used for the dispersal of cerumen, some commercially available. Saxby and colleagues ${ }^{1}$ have attempted to identify the ideal cerumenolytic, at first by in vitro methods. They conclude that aqueous solutions disperse cerumen best, with distilled water being as good as any other liquid. Oil-based solutions had very little cerumenolytic activity at all.

The external auditory canal is a breeding ground for many micro-organisms, some harmless and some pathogenic. Lambor and colleagues ${ }^{2}$ review the presentation and management of patients with necrotising otitis externa. Most of these patients are elderly diabetics, and Pseudomonas aeruginosa remains the commonest organism. There is no firm consensus on management of this condition, with cases tending to be managed on an individual basis. There is still a role for surgical debridement in some cases, although most patients respond to targeted and prolonged antipseudomonal chemotherapy. Other organisms are carried in the external auditory canal and are not clinically pathogenic. They can, however, potentially transmit infection. Eftekharian and colleagues ${ }^{3}$ demonstrate that hepatitis B virus DNA can be routinely isolated from the cerumen of hepatitis B infected patients. Thus, cerumen can be a potential source of transmission of hepatitis B virus.
Another area of controversy concerning the potential transmission of infective material is that of Creutzfeldt-Jakob disease (CJD). This disease has fluctuated in prominence in the public eye and is reviewed by Sethi and colleagues. ${ }^{4}$ In the past, precautions to minimise the transmission of CJD have lacked an evidence base. This review gathers together all current evidence in order to propose simple precautions which can be introduced into everyday practice.

Many of us in the United Kingdom have been aware of changing social patterns, particularly among teenagers and young adults. Many of these changes have been a product of readily available alcohol. One such change is that young women are now much more likely to be involved in non-domestic violence. Trinidade and colleagues ${ }^{5}$ have undertaken a multicentre study which demonstrates an 825 per cent increase in nasal fractures in females aged 13-20 years between 2002 and 2009. They propose that this change can be attributed to a rise in so-called 'ladette culture'.

ROBIN YOUNGS EDWARD FISHER Editors

References

1 Saxby C, Williams R, Hickey S. Finding the most effective cerumenolytic. J Laryngol Otol 2013;127:1067-70

2 Lambor DV, Das CP, Goel HC, Tiwari M, Lambor SD, Fegade MV. Necrotising otitis externa: clinical profile and management protocol. J Laryngol Otol 2013;127:1071-7

3 Eftekharian A, Moghaddasi H, Gachkar L, Amlashi SSA. Detection of hepatitis B virus in the cerumen of patients with chronic hepatitis B infection. J Laryngol Otol 2013;127: 1065-6

4 Sethi N, Kane J, Condon L. Creutzfeldt-Jakob disease and ENT. J Laryngol Otol 2013;127:1050-5

5 Trinidade A, Buchanan MA, Farboud A, Andreou Z, Ewart S, Mochloulis $\mathrm{G}$ et al. Is there a change in the epidemiology of nasal fractures in females in the UK? J Laryngol Otol 2013; 127:1084-7 